\section{Primary mediastinal giant teratocarcinoma}

D ear Sir,

A 25-year-old male presented with right hemi-thoracic chest pain, brassy cough, and progressively worsening dyspnoea for last two months. He developed facial puffiness and edema of both the upper limbs for last one month.

Examination revealed mild pallor, distended and nonpulsatile jugular veins, and bilateral gynaecomastia. Other general examination was normal.

Chest examination revealed bulging right thoracic wall with ipsilateral reduction of respiratory movements. The same areas were dull on percussion with marked reduction of vocal fremitus, vocal resonance and breath sounds. D e-spine sign was positive up to T8 vertebra. Cardiac examination was normal except for shift of cardiac apex $3 \mathrm{~cm}$ lateral to mid clavicular line in $5^{\text {th }}$ intercostal space on left side. Abdomen including genitalia, and nervous system examination were normal.

Skiagram chest PA view revealed homogeneous opacification of right hemi thorax with obliteration of right costophrenic and cardiophrenic angles, and right border of heart. There was mediastinum shift towards left side [Figure 1].

Contrast enhanced CT scan chest revealed a huge $(20.8 \times 13.0 \times 16.0 \mathrm{~cm})$ heterogeneously enhancing soft tissue density mass lesion in the anterior mediastinum extending from the thoracic inlet almost till the diaphragm occupying nearly the entire right hemi-thorax with extension across the midline to left. This mass was causing severe compression of the mediastinal vessels and bronchial orifices [R ight> left]. The cardiac silhouette was also markedly pushed to the left side [Figure 2]. Moderate

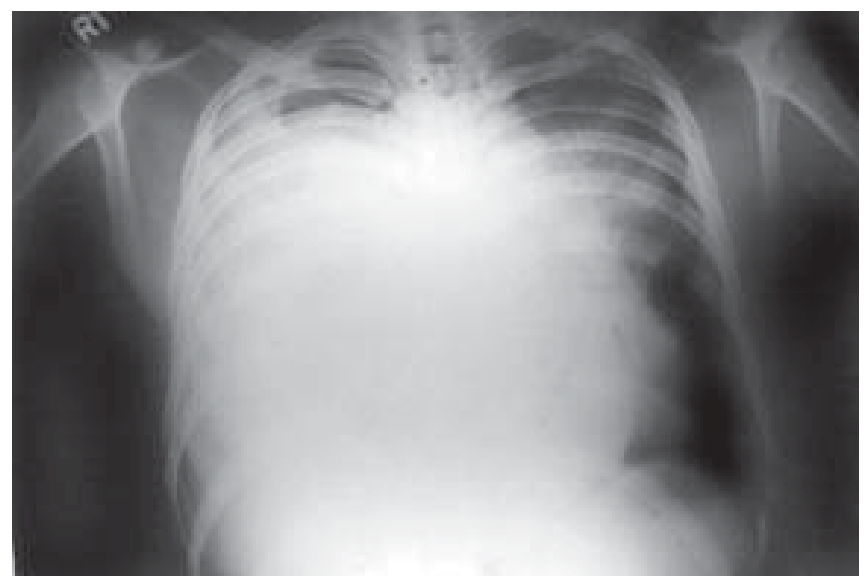

Figure 1: Radiograph chest PA view showing near complete homogenous opacification of right hemi-thorax with mediastinal shift towards left side

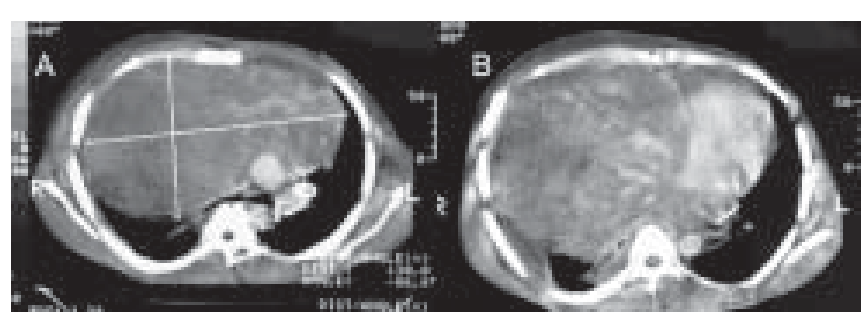

Figure 2: (A and B) CECT images showing well defined soft tissue density mass with patchy enhancement in the anterior mediastinum in right hemi-thorax with extension across the midline to left. Severe compression with infiltration of the mediastinal vessels and bronchial orifices [Right>left] is seen which are pushed posteriorly and to the left. The cardiac silhouette also seen displaced to the left side

amount of pleural fluid was also seen on right side.

$\mathrm{H}$ emorrhagic pleural fluid was aspirated with plenty of malignant cells. USG guided trucut biopsy of the mass was diagnostic of teratocarcinoma.

Extragonadal germ cell tumors are uncommon nonetheless the anterior mediastinum is the most common extragonadal site. Mostly mediastinal teratomas $(>75 \%)$ are benign, and have equal incidence in men and women, but $90 \%$ of malignant tumors are seen in men. ${ }^{[1]}$

$M$ ature teratomas demonstrate a mixture of tissues from one or more of the germinal layers, i.e. ectoderm, endoderm, and mesoderm. If the tumor contains any immature or embryonal structures, it is considered an immature teratoma. A mature teratoma may rarely undergo malignant transformation of one of the mature elements. The term teratocarcinoma is used for the combination of teratoma plus embryonal carcinoma.

Review of literature regarding the size of teratocarcinoma mentions size ranging from 4 to $13 \mathrm{~cm}$ in diameter. ${ }^{[2,3]}$ In our patient, the size of the tumor $(20.8 \times 13.0 \times$ 
$16.0 \mathrm{~cm}$ ) was even beyond the largest size reported.

Clinically teratocarcinomas may be asymptomatic or present with symptoms referable to compression of mediastinal structures such as dyspnoea, cough, dysphagia or chest pain. In larger tumors, signs of superior vena cava obstruction may occur. U ncommon presentations include rupture into the pleural space, tracheo-bronchial tree, lung parenchyma and pericardial $\mathrm{sac}^{[4]}$ In some cases gynaecomastia and rarely hyperthyroidism (due to raised $\beta \mathrm{HCG}$ ) may be the only indicators to the tumor. ${ }^{[1]}$

Plain radiographic findings of teratomas are non-specific. On CT scan the combination of fluid, fat, calcification, and soft-tissue attenuation in an anterior mediastinal mass is highly specific of teratomas. A malignant teratocarcinoma may be indistinguishable from mature teratoma but presence of more solid components and aggressive features like chest wall invasion, pulmonary/ liver metastases in association with increased tumor markers is very suggestive of malignancy. ${ }^{[5]}$

Singhal M, Jhavar $\mathbf{D}^{1}$ Department of Radio Diagnosis, PGIMER, Chandigarh-160 012, India. 'Department of Internal Medicine, MGM Medical College and MY Hospital, Indore, MP- 452001, India.

Correspondence to: Dr. Manphool Singhal, E-mail: drmsinghal@yahoo.com

\section{References}

1. Anuradha S, Singh NP, Agarwal SK, Gondal R, Prakash A, et al. Primary retroperitoneal metastatic embryonal carcinoma. J Indian Acad Clin Med 2006;2:210-3.

2. Murakawa T, Nakajima J, Kohno T, Tanaka M, Takeuchi E, Oka T, et al. Primary mediastinal germ cell tumor: Report of a case. Kyobu Geka 2000;53:345-9.

3. Iwasaki T, Luchi K, Matsmura A, Sueki H, Yamamato S, Mori T. Intrapulmonary mature teratoma. Jpn J Thorac Cardiovasc Surg 2000;48:468-72.

4. Sakamoto K, Kase M, Mo M, Kurata H. Mediastinal mature teratoma perforated into the pericardial sac: A case report. Kyobu Geka 2000;53:74-7.

5. Lee JK, Sagel SS, Stanley RJ, Heiken JP. Computed body tomography with MRI correlation. $3^{\text {rd }}$ ed. Vol. 1. p. 265-6. 